Proceedings of the 47th International School and Conference on the Physics of Semiconductors "Jaszowiec 2018"

\title{
Effect of Reservoirs on Transport Properties of Doped Structures*
}

\author{
K. Kulinowski, M. WoŁoszyn and B.J. SpisaK ${ }^{\dagger}$ \\ AGH University of Science and Technology, Faculty of Physics and Applied Computer Science, \\ al. A. Mickiewicza 30, 30-059 Kraków, Poland
}

\begin{abstract}
Influence of boundary conditions on the transport properties of a semiconductor device is presented. The boundary conditions are generated by convolution of the supply function and Lorentzian or Voigt profile. Transport characteristics are determined by solution of the kinetic equation within the relaxation time approximation.
\end{abstract}

DOI: 10.12693/APhysPolA.134.923

PACS/topics: 51.10.+y, 72.10.Bg, 72.80.Ey

\section{Introduction}

It is generally accepted that electronic devices can be treated as open systems because they exchange both carriers and energy with connected contacts. Therefore, a theoretical description of transport properties of such systems requires some methods developed for analysis of nonequilibrium states in the classical or quantum phase space of position and momentum variables. Such possibility is created by the kinetic method based on the integro-differential equation in the form [1]:

$$
\begin{aligned}
& \frac{\partial \varrho(x, p, t)}{\partial t}+\frac{p}{m^{*}} \frac{\partial \varrho(x, p, t)}{\partial x} \\
& -\int \mathrm{d} p^{\prime} W\left(x, p-p^{\prime}\right) \varrho\left(x, p^{\prime}, t\right)=I[\varrho(x, p, t)],
\end{aligned}
$$

where $m^{*}$ is the effective mass of carriers, $\varrho(x, p, t)$ is the Wigner distribution function, $I[\varrho(x, p, t)]$ is the collision integral, and $W(x, k)$ is the nonlocal potential. Its form is given by the formula

$$
\begin{gathered}
W\left(x, p-p^{\prime}\right)=\frac{1}{2 \pi \mathrm{i} \hbar} \int \mathrm{d} X\left[U\left(x+\frac{X}{2}\right)\right. \\
\left.-U\left(x-\frac{X}{2}\right)\right] \exp \left(-\frac{\mathrm{i}\left(p-p^{\prime}\right) X}{\hbar}\right),
\end{gathered}
$$

where $U(\xi)$ represents the potential energy of the carriers. The transport equation given by Eq. (1) is usually solved with the inflow boundary conditions determined by the Fermi-Dirac distribution function or the supply function, i.e. the Fermi-Dirac function integrated over the transverse momentum [2]. However, a modified form of the inflow boundary conditions has been recently proposed in Ref. [3]. Results of this research clearly prove that the transport properties of active region of a nanodevice cannot be analyzed independently of the contacts, because the thermalization effect due to scattering in contacts strongly reduces the electronic current through the \footnotetext{
2018

*The text of Acknowledgements was corrected on December 28,

${ }^{\dagger}$ corresponding author; e-mail: bjs@agh.edu.pl
}

considered nanosystem, regardless of the dissipative flow of conduction electrons which is included in Eq. (1).

In this report we present a modified form of the supply function which allows one to include the scattering processes in the contacts and we solve the stationary form of Eq. (1) in the classical limit using the proposed boundary conditions. We also determine some transport characteristics of homogeneous semiconductor device made of III$\mathrm{V}$ compound in CPP geometry and we investigate an influence of the boundary conditions on the characteristics.

\section{Theory and model of the system}

In the classical limit, the Wigner function can be approximated by the classical distribution function $f(x, p, t)$ according to the formula [4]:

$$
\begin{aligned}
& \int_{\Delta x \Delta p} \mathrm{~d} x \mathrm{~d} p \varrho(x, p, t)= \\
& \quad \int_{\Delta x \Delta p} \mathrm{~d} x \mathrm{~d} p f(x, p, t)+\mathrm{O}\left[\left(\frac{2 \pi \hbar}{\Delta x \Delta p}\right)^{2}\right] .
\end{aligned}
$$

In turn, the application of the gradient approximation to the nonlocal potential given by Eq. (2) allows us to reduce Eq. (1) to the Boltzmann form, namely

$$
\begin{aligned}
& \frac{\partial f(x, p, t)}{\partial t}+\frac{p}{m^{*}} \frac{\partial f(x, p, t)}{\partial x}-\frac{\partial U(x)}{\partial x} \frac{\partial f(x, p, t)}{\partial p}= \\
& I[f(x, p, t)] .
\end{aligned}
$$

The scattering integral is modeled within the relaxation time approximation [5], i.e.

$$
I[f(x, p)]=-\frac{f(x, p)-f^{0}(x, p)}{\tau},
$$

where $f^{0}(x, p)$ is the equilibrium distribution function, $\tau$ is the relaxation time which characterizes the elastic scattering processes of carriers on a set of randomly distributed $\delta$-function dopants in the active region of the device, within the assumed Gaussian model of disorder. It means that the average disorder potential is equal to zero, and the second cumulant of the potential - the potential correlator is constant. Taking into account this assumption we can write down the stationary Boltzmann equation in the form 


$$
\frac{p}{m^{*}} \frac{\partial f(x, p)}{\partial x}=-\frac{f(x, p)-f^{0}(x, p)}{\tau} .
$$

This equation is solved with the following inflow boundary conditions [6]:

$$
\begin{aligned}
& \left.f(x=0, p)\right|_{p>0}=f_{\text {therm }}^{L}(E(p)), \\
& \left.f(x=L, p)\right|_{p<0}=f_{\text {therm }}^{R}(E(p)),
\end{aligned}
$$

where $f_{\text {therm }}^{L(R)}(E(p))$ are thermalized distribution functions for the left $(L)$ and right $(R)$ contacts (as shown in Fig. 1) in the form

$$
f_{\text {therm }}^{L(R)}(E(p))=\int_{0}^{\infty} \mathrm{d} E \delta_{\Gamma}(E-E(p)) f^{L(R)}(E),
$$

where $E(p)$ is the quadratic dispersion relation, $\delta_{\Gamma}(E)$ is represented by a $\Gamma$-parametrized Lorentzian or Voigt profile with a width proportional to the phase-breaking strength $\Gamma=\hbar / 2 \tau_{\Gamma}$, whereby $\tau_{\Gamma}$ is the relaxation time for the scattering processes included in the equilibrium distribution. The supply function $f^{L(R)}(E)$ is given by the formula [2]:

$$
\begin{aligned}
& f^{L(R)}(E)= \\
& \quad \frac{m^{*} k_{\mathrm{B}} T}{\pi \hbar^{2}} \ln \left(\exp \left(-\left(E-\mu_{F}^{L(R)}\right) / k_{\mathrm{B}} T\right)+1\right),
\end{aligned}
$$

where $k_{\mathrm{B}}$ is the Boltzmann constant, $T$ is the temperature, and $\mu_{F}^{L(R)}$ is the electrochemical potential in the left (right) contact. The difference between the electrochemical potentials of the left and right reservoirs corresponds to the applied bias voltage between them, i.e., $e V_{B}=\mu_{F}^{L}-\mu_{F}^{R}$.

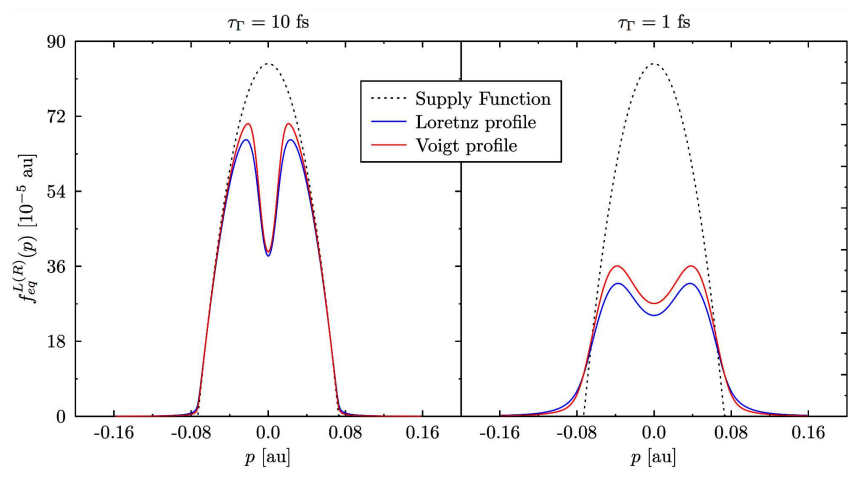

Fig. 1. Lorentz and Voigt profiles compared to the supply function for different relaxation times $\tau_{\Gamma}$.

We consider a model of electronic device for which an active region is made of semiconductor compound GaAs material. The active region is connected to two contacts with potential difference $V_{B}$. Scheme of the considered system is shown in Fig. 2. Simulations were performed with the following material and simulation parameters: device length $L=1 \mu \mathrm{m}$, contact temperature
$T=77 \mathrm{~K}$, the Fermi level in right contact $\mu_{F}^{R}=0.08 \mathrm{eV}$, electron band mass $m^{*} / m_{0}=0.067$, the relaxation time inside simulation domain $\tau=300 \mathrm{fs}$, maximum momentum $p_{\max }=0.16$ a.u., number of steps $N_{x}=100$ and $N_{p}=60$.

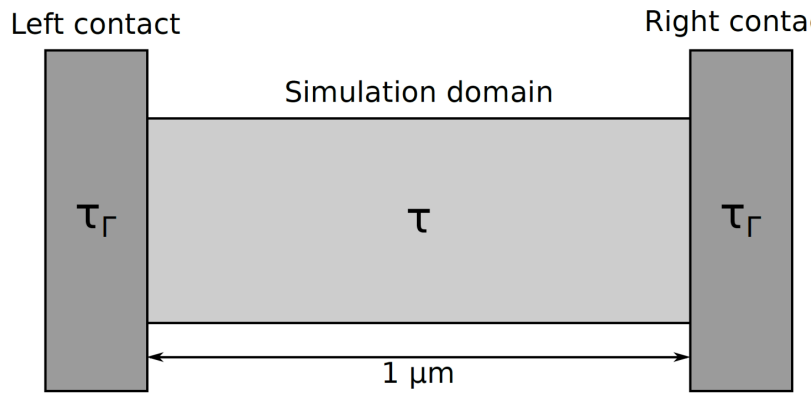

Fig. 2. Scheme of the device which is modeled as an open system. Effect of the contacts is characterized by the relaxation time $\tau_{\Gamma}$, and the active region of the device is characterized by the relaxation time $\tau$.

\section{Results and discussion}

For a given voltage value $V_{B}$, we determine the distribution function $f\left(x, p ; V_{B}\right)$ by solving Eq. (6) with boundary conditions ( 7$)$. Then we calculate the current as the first moment of the distribution function in the following way:

$$
I\left(V_{B}\right)=\frac{1}{2 \pi L} \int \mathrm{d} x \int \mathrm{d} p \frac{p}{m^{*}} f\left(x, p ; V_{B}\right) .
$$

Owing to that we can determine the current-voltage characteristics. Results of our calculations based on the considered theoretical scheme are presented in Fig. 3. We can see that for the relaxation time $\tau_{\Gamma}=10$ fs the $I-V$ characteristics are nearly identical, but for $\tau_{\Gamma}=1$ fs the difference between them is visible.

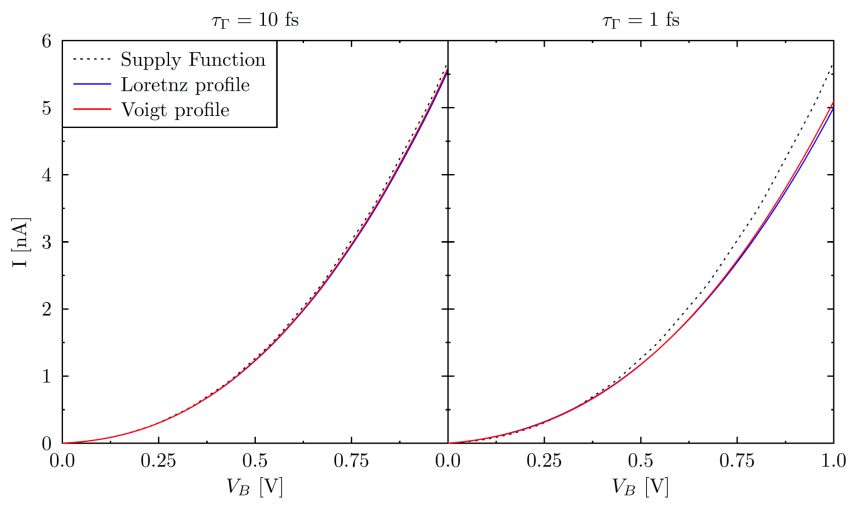

Fig. 3. Current-voltage characteristics for different relaxation times in contacts $\tau_{\Gamma}$ and a fixed value of relaxation time $\tau$ in active element of the device, when the Lorentzian and Voigt profile are used.

These results reflect the fact that the Lorentzian and Voigt profiles which determine the inflow boundary conditions (cf. Fig. 1) differ slightly from each other. Mini- 
mum of the profiles near $p=0$ has little influence on the current because of its small weight in integral given by Eq. (10), while the tails of the distribution function at boundaries give much larger contribution.

Figure 4 displays the relative current change as a function of relaxation time $\tau_{\Gamma}$, where $\Delta I\left(\tau_{\Gamma}\right)=I_{0}-I\left(\tau_{\Gamma}\right)$, and $I_{0}$ is the current calculated with the supply function as boundary condition.

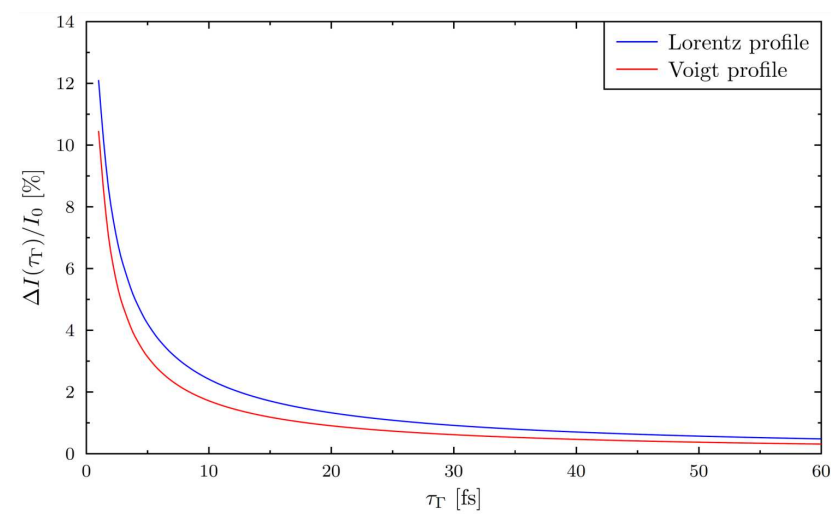

Fig. 4. Relative current change due to scattering processes in contacts, for voltage $V_{B}=1 \mathrm{~V}$.

For both used profiles, the relative change of current is similar, i.e., increasing with decreasing $\tau_{\Gamma}$. However, for the Lorentz profile the change is larger, especially at smaller relaxation times $\tau_{\Gamma}$. As it should be expected, the asymptotic behavior when $\tau_{\Gamma} \rightarrow 0$ leads to rapid increase in resistance of contacts, while for $\tau_{\Gamma} \rightarrow \infty$ the resistance vanishes and the current is equal to $I_{0}$.

\section{Conclusions}

Thermalization of electrons in contacts due to scattering processes slightly changes the electronic transport in the diffusion limit. The difference between relative change of the current when the Lorentz or Voigt profile is used for boundary condition is up to several percent depending on the relaxation time in the contacts.

\section{Acknowledgments}

K.K. has been partly supported by the EU Project POWR.03.02.00-00-I004/16 and by the AGH UST dean grant No. 05.11.220.717/45 for Ph.D. students and young researchers within subsidy of the Ministry of Science and Higher Education. B.S. and M.W. have been partially supported by the Faculty of Physics and Applied Computer Science AGH UST statutory tasks within subsidy of Ministry of Science and Higher Education.

\section{References}

[1] D. Querlioz, P. Dollfus, The Wigner Monte Carlo Method for Nanoelectronic Devices, ISTE and Wiley, London 2010.

[2] D.K. Ferry, S.M. Goodnick, J. Bird, Transport in Nanostructures, Cambridge University Press, Cambridge 2009 .

[3] M. Wołoszyn, B.J. Spisak, Phys. Rev. B 96, 075440 (2017).

[4] D. Szydłowski, M. Wołoszyn, B.J. Spisak, Semicond. Sci. Technol. 28, 105022 (2013).

[5] N.W. Ashcroft, N.D. Mermin, Solid State Physics, Holt Rinehart and Winston, New York 1976.

[6] W.R. Frensley, Rev. Mod. Phys. 62, 745 (1990). 